\title{
MODELO PARA MEDIC̄ÃO DO FLUXO DE CAIXA EM RISCO: APLICAÇÃO A DISTRIBUIDORAS DE ENERGIA ELÉTRICA
}

\section{RESUMO}

O gerenciamento de riscos é um assunto que assume papel relevante no ambiente das instituições não financeiras. A despeito da importância crescente do assunto, discussões acerca da implementação de um modelo capaz de avaliar os riscos aos quais os fluxos de caixa das empresas estão expostos ainda são incipientes. Considerando a existência dessa lacuna e a importância do tema para as empresas, este estudo propõe a construção de um modelo teórico de fluxo de caixa em risco (ou cashflow-at-risk) e o aplica a empresas pertencentes ao setor de distribuição de energia elétrica no Brasil. Tal modelo deve ser capaz de informar a probabilidade de uma empresa ter um fluxo de caixa livre negativo em suas datas de pagamento.

\section{Fernanda Finotti Cordeiro Perobelli}

USP

José Roberto Securato

USP

ABSTRACT Risk management has been taking an important role in the environment of non-financial businesses. However devel opment of a model capable of evaluating risks affecting those companies is still wanting. Considering the gap, the article sets as its objective to develop an empirical cash flow at risk model applied to companies operating in electricity distribution. The model is supposed to supply information on the probability of funds to meet its accounts payable at a given date or the probability of having a negative free cash flow on its payment dates.

PALAVRAS-CHAVE Fluxo de caixa em risco, instituições não financeiras, distribuidoras de energia elétrica.

KEMORDS Cashflow-at-risk, non-financial institutions, electricity sector distributors. 


\section{INTRODUÇÃO}

O gerenciamento de riscos de mercado, crédito, operacionais ou legais é um assunto que assume papel relevante e definitivo no ambiente das instituições financeiras. M ais recentemente 0 assunto vem ganhando espaço também no âmbito de instituições não financeiras. Para estas, é igualmente importante saber o risco de não-pagamento inerente aos financiamentos concedidos a seus clientes (risco de crédito), o risco advindo de fal has humanas dentro da organização (risco operacional), o risco de ser acionada legalmente por seus stakeholders (funcionários, credores, concorrentes, clientes e comunidade) e, de grande importância para o gerenciamento de seu fluxo de caixa, os riscos de mercado, aqui entendidos como variações nos preços formados no âmbito do mercado financeiro - juros, câmbio, commodities - , flutuações na demanda do mercado consumidor e na oferta dos insumos utilizados no processo produtivo, capazes de afetar sua capacidade financeira e solvência.

Especificamente com relação às empresas brasileiras, Castro (2002) destaca que a estabilização econômica obtida a partir de 1994, que possibilitou o planejamento de longo prazo, e a abertura econômica, que propiciou a inserção de forma mais ampla dessas empresas no contexto internacional - ao mesmo tempo em que as expôs a novas fontes de risco - , são fatores que colaboram para a necessidade de se desenvolver um controle eficaz de riscos de mercado dentro das empresas.

Dentre os benefícios advindos da implantação de sistemas de medição e gerenciamento de riscos de mercado no âmbito das instituições não financeiras, destacam-se como os mais diretos: o controle dos fluxos de caixa necessários ao cumprimento dos compromissos assumidos pela empresa, que incluem 0 pagamento de fornecedores, despesas operacionais e financeiras, amortização de empréstimos, e dos investimentos programados; a redução da volatilidade desses fluxos e, conseqüentemente, da probabilidade de a empresa deixar de honrar compromissos futuros. Benefícios adicionais incluem o aumento da transparência aos investidores, a rápida assimilação de novas fontes de riscos de mercado pelos gestores e, especificamente no caso brasileiro, a adequação antecipada da empresa à regulação. A Comissão de Valores Mobiliários (CVM), por meio do Ofício Circular 01/2002 de 14 de janeiro de 2002, reforçou a necessidade de se divulgarem os riscos de mercado incorridos pelas empresas em seus demonstrativos financeiros.
A despeito da importância crescente do assunto, discussões acerca da implementação de um modelo capaz de avaliar, de forma conjunta, os riscos de mercado aos quais as empresas estão expostas ainda são incipientes. Considerando a existência dessa lacuna e a importância do tema para as empresas, este estudo tem como objetivo principal a proposição de um modelo teórico para a mensuração do fluxo de caixa em risco das empresas e, como objetivo secundário, sua aplicação empírica a distribuidoras de energia elétrica no Brasil. Tal modelo deve ser capaz de informar a probabilidade de uma empresa não dispor de recursos ou apresentar fluxo de caixa livre (FCF) negativo para honrar seus compromissos em determinadas datas futuras de pagamento ou vértices do fluxo.

\section{REFERENCIAL TEÓRICO}

O uso de sistemas quantitativos para a medição de riscos de mercado começou a se difundir entre instituições financeiras a partir de 1994, com o lançamento, pelo JP Morgan, do documento RiskM etrics, que trazia, entre outras coisas, a metodologia de cálculo do Value-at-Risk (VaR). Dois anos se passaram até que a Securities and Exchange Comission (SEC), por pressões de órgãos reguladores, propusesse em 28 de dezembro de 1995 uma regra que exigia das companhias americanas informações quantitativas sobre riscos de mercado (Linsmeier e Pearson, 1997).

Tal regulação, aliada a um cenário de volatilidade crescente - como a proporcionada pela crise asiática em outubro de 1997 e a crise russa em agosto de 1998 -, serviu como incentivo para que muitas empresas americanas passassem a tentar adaptar o instrumento de controle de riscos de mercado até então utilizado apenas por instituições financeiras ( $V a R$ ) às suas necessidades. Contudo, apesar de simples, intuitivo e aceito pelo mercado e pelas instituições reguladoras, tentativas mais robustas de adaptação do VaR ao ambiente corporativo foram revelando certas deficiências dessa medida em relação ao controle de todos os riscos de mercado a que estão expostas empresas não financeiras. Tais deficiências fizeram com que novas medidas de risco de mercado fossem ganhando espaço no âmbito dessas empresas.

Tentativas rudimentares de verificação dos impactos de oscilações nos preços de mercado sobre o fluxo de caixa são atribuídas a Vermeulen (1994), Shapiro e Titman (1999), e Bauman, Saratore e Liddle (1999). 
Esses autores, entretanto, não chegaram a sugerir um model o completo, tratan do a questão apenas de forma geral. Provavelmente, um dos trabalhos pioneiros e mais detal hados no cál culo do fluxo de caixa em risco foi o desenvolvido por Hayt e Song (1995), que propunha uma medida de sensibilidade dos fluxos de cai$x a$ a fatores de risco. Tal medida buscava relacionar a probabilidade de a empresa atingir determinado nível de fluxo de caixa que a impedisse de honrar seus compromissos e programações de investimentos, com mudanças em preços financeiros em determinado período de tempo. Posteriormente, o trabalho de Stulz e Williamson (1997) também mencionou a possibilidade de utilizar a simulação para a obtenção da distribuição esperada dos fluxos de caixa futuros.

Entretanto, o refinamento da medição de fluxos de caixa em risco só ocorreu em 1999, com a elaboração do CorporateM etrics Technical Document (RiskM etrics Group, 1999). O foco desse documento estava nos potenciais impactos de mudanças nas taxas de mercado sobre os resultados financeiros da empresa em um intervalo de tempo t. Entre as medidas de risco propostas e analisadas pelo CorporateM etrics estava 0 Cash-Flow-at-Risk (C-FaR). A metodologia empregada para o cálculo dessa medida tomava emprestados conceitos utilizados para o cálculo do VaR, adaptando-os ao ambiente corporativo, e estendia a tradicional técnica de análise de sensibilidade além de uns poucos cenários extremos, considerando um amplo conjunto de cenários simulados. Para a elaboração do método proposto, seria necessário estimar relações econométricas entre os fatores de risco e a variável de interesse (fluxo de caixa). Depois de determinadas tais relações, passar-se-ia à investigação do modelo capaz de descrever o comportamento dos fatores de risco. Para tal, o documento determinava não apenas que fosse construído um modelo capaz de descrever tão corretamente quanto possível a evolução dos fatores de risco, mas que ele também fosse consistente com teorias econômicas rel evantes. A sugestão do documento era a utilização dos chamados Vetores Auto-Regressivos (Vector Autoregressive Model ou VARM), nos quais o valor de cada variável depende não só de seus valores passados, mas também dos valores passados de todas as outras variáveis do sistema, o que permiti ria a previsão conjunta da média condicional dos fatores de risco.

Em 2000 o modelo desenvolvido pela consultoria $N$ ational Economic Research Associates (NERA) abandonou o enfoque de séries de tempo e bottom-up (da identificação do comportamento dos fatores de risco para o fluxo de caixa em risco), predominante nos modelos anteriormente apresentados, propondo uma modelagem tipo top-down (da observação agregada do fluxo de caixa de um conjunto de empresas para o fluxo de caixa em risco de cada uma delas). A medida proposta pela NERA - denominada Comparables CashFlow-at-Risk (C-FaR) - seria obtida a partir da distribuição de probabilidades de fluxos de caixa observados diretamente e não mais via distribuição dos fatores de risco. Para tanto, seria necessário aglutinar os fluxos observados em um conjunto amplo e homogêneo de empresas. Depois de estimada tal distribuição, ela poderia ser usada para gerar uma série de estatísticas, tais como os percentis de $5 \%$ e $1 \%$ da cauda inferior da distribuição. Portanto, a partir de tal distribuição seria possível responder a perguntas do tipo: "se uma empresa tem características que a classificam nesta amostra específica, qual percentual de queda máxima no fluxo de caixa tal empresa pode experimentar no horizontet, com 95\% de confiabilidade?" (Stein, Usher, LaG attuta e Youngen, 2001).

Por fim, recentemente no mercado brasileiro, a RiskControl, em parceria com a Consultoria Tendências, desenvolveu um modelo que considerava cenários probabilísticos para os fatores de risco construídos a partir da integração de metodologias estatísticas e model os macroeconômicos estruturais ( LaRoque et al., 2003). Os modelos econômicos estruturais fazem uso da estrutura sugerida pela teoria macroeconômica na formulação de suas previsões. As relações podem ser expressas por equações, estimadas via modelos econométricos, que se articulam. Assim, é possível captar as interações rel evantes entre as variáveis de interesse.

Diferentemente do VARM, no entanto, esses modelos assumem a existência de variáveis independentes exógenas nas equações, estimadas por meio de dados históricos e regressões econométricas, e variáveis dependentes endógenas, que são função das exógenas estimadas. Para inserir incerteza no modelo, a RiskControl/Tendências propunha que as séries de variáveis exógenas fossem decompostas em tendência e resíduo. Estimadas as tendências das séries, a volatilidade condicional de cada uma delas seria estimada usando-se as diferenças entre os valores assumidos pela série e sua ten dência (resíduo). Entendida a dinâmica da volatilidade individual de cada uma das variáveis exógenas, seria preciso verificar a dinâmica de covariância entre elas. De acordo com a RiskControl/Tendências, estas seriam melhor estimadas pela tendência das séries e não pelos resíduos. Estimadas as vola- 
tilidades e as covariâncias, a simulação das trajetórias conjuntas assumidas pelas variáveis exógenas seria obtida via Simulação de M onte Carlo. Quanto às endógenas, se a incerteza em relação às exógenas já estivesse corretamente modelada, bastaria substituir os valores simulados para as exógenas nas equações das endógenas.

Identificados os modelos de medição do fluxo de caixa em risco existentes, para o desenvolvimento do presente modelo foram combinadas as principais contribuições já dadas sobre 0 assunto com pontos ainda não suficientemente explorados, de modo a obter-se um modelo empiricamente viável para o gerenciamento do fluxo de caixa em risco das empresas brasileiras.

\section{MODELO TEÓRICO PARA CASHFONAAT-RISK}

Este tópico apresenta um model o teórico para a mensuração do fluxo de caixa em risco das empresas, aqui entendido como a probabilidade de a empresa não dispor de recursos para honrar seus compromissos em determinadas datas futuras (vértices do fluxo) ou, estatisticamente, como o percentil associado à estatística de ordem zero da distribuição do fluxo de caixa livre da empresa. Ressalte-se novamente que o model o aqui proposto combina as principais contribuições já dadas sobre o assunto - em especial, as fornecidas pelo CorporateM etrics Technical Document - com pontos ainda não suficientemente explorados na literatura.

Metodologicamente, para a construção do modelo foram considerados os pontos seguintes: (1) definir as variáveis de estudo, subdividindo-as em variáveis dependentes (fluxo de caixa) e candidatas a variáveis independentes (fatores de risco macroeconômicos e próprios do negócio); (2) definir os vértices temporais em que tais variáveis serão observadas e o horizonte temporal de previsão (número de passos à frente); (3) identificar os fatores de risco relevantes (entre macroeconômicos e próprios da empresa) via estimação da relação estatística existente entre a variável dependente e as candidatas a variáveis independentes; (4) sugerir um tratamento para o gerenciamento dos fatores de risco próprios e estimar, via modelagem econométrica, o comportamento médio esperado dos fatores de risco macroeconômicos, bem como sua matriz de variância-covariância; (5) simular cenários para os fatores de risco macroeconômicos no horizonte de previsão, tomando o cuidado de manter a estrutura de variância-covariância observada entre as séries histó- ricas de fatores; (6) inserir os valores previstos para os fatores de risco em cada cenário na equação que relaciona o comportamento de tais fatores ao comportamento da variável dependente (fluxo de caixa); (7) montar a distribuição simulada da variável dependente (fluxo de caixa) e determinar a estatística de interesse de tal distribuição.

\section{Variáveis do modelo, seus vértices de medição e seu horizonte de previsão}

Para a formulação do modelo, é preciso definir a variável de interesse (variável dependente), assim como selecionar candidatas a variáveis independentes - fatores de risco macroeconômicos e próprios do negócio, capazes de alterar o comportamento da variável dependente ao longo do tempo.

Considerando-se que o modelo proposto baseia-se na mensuração do fluxo de caixa em risco, a variável de interesse proposta neste trabal ho é o fluxo de caixa livre da empresa, observado em certas datas de pagamento futuras, ou vértices do fluxo. Há, entretanto, que se escolher uma proxy para tal fluxo. Contabilmente, 0 fluxo de caixa de uma empresa é determinado conforme apresentado no Quadro 1.

No Quadro 1, o fluxo de caixa após as atividades de financiamento constitui o fluxo de caixa à disposição da empresa. N este artigo, tal fluxo é denominado Fluxo de Caixa Livre (FCF), constituindo-se a variável de interesse (ou variável dependente) do modelo de mensuração do fluxo de caixa em risco.

As candidatas a variáveis independentes são fatores capazes de provocar oscilações no fluxo de caixa livre das empresas, ou fatores de risco. Tais fatores de risco podem ser macroeconômicos (nível de taxas de juros, câmbio, inflação, risco-país, produção nacional) - capazes de afetar, em maior ou menor grau, todas as empresas da economia - e fatores de risco próprios do negócio (nível de endividamento, mercado atendido, investimentos programados). No modelo proposto, os candidatos a fatores de risco macroeconômicos são indicados pela letra $m$ e os candidatos a fatores de risco próprios pela letra f.

A consideração no modelo de características próprias da empresa como candidatas a fatores de risco tem como objetivo dar-Ihe flexibilidade. Enquanto as oscilações nos fatores de risco macroeconômicos não podem ser determinadas a priori pela empresa, mas apenas estimadas e hedgeadas caso seus potenciais efeitos sobre o fluxo de caixa assim o justifiquem, a identificação prévia de fatores de risco gerenciáveis per- 
mite à empresa verificar, por exemplo, quais seriam os impactos em seu fluxo de caixa em determinada data se, além de choques esperados nos fatores de risco macroeconômicos, ela também decidisse al terar sua estrutura operacional e financeira via aumento de market share, novos investimentos, aumento de endividamento, substituição de dívida em moeda estrangeira por dívida em moeda nacional, etc.

Com relação aos vértices de medição, datas em que se observam as variáveis dependentes e independentes, este estudo propõe a utilização de vértices trimestrais. Dessa forma, tal como na estimação do VaR, em que se assume que pagamentos e recebimentos não ocorram todos os dias, mas apenas em determinadas datas, neste model o os fluxos de caixa da empresa apenas são observados a cada trimestre, vértices que concentram ( hipoteticamente) todos os pagamentos e recebimentos futuros.

Ressal te-se que a divulgação pelas empresas de dados contábeis em base trimestral é a responsável por tal escolha. Obviamente, tal restrição é relevante apenas ao pesquisador externo. Caso o estudo esteja sendo conduzido com informações privadas, tais vértices podem ser mensais, ou obedecer às datas constantes no budget plan elaborado pela empresa.

Da mesma forma, o horizonte de previsão - número de passos à frente em que os fluxos são observados - fica a critério do pesquisador e da empresa interessada. Ressalte-se, porém, que, quanto mais longo for o prazo, menor a confiabilidade das estatísticas geradas. $\mathrm{Nada}$ impede, entretanto, que as estimativas geradas sejam periodicamente revistas, de modo a se atual izar constan- temente a estimação pela consideração de novas observações passadas.

\section{Identificação dos fatores de risco macroeconômicos e próprios relevantes}

Enquanto a metodologia do VaR exige a identificação de um número reduzido de fatores de risco primitivos que repliquem, de forma linear e determinística, as oscilações no valor de mercado dos ativos originais, identidades semelhantes não são facilmente obtidas para o cálculo do fluxo de caixa em risco. No caso do fluxo de caixa em risco, o mais próximo da replicação determinística utilizada pelo VaR a que se chega é via estimação de equações que relacionem as oscilações no fluxo de caixa livre da empresa (variável dependente) a oscilações nos fatores de risco (variáveis independentes).

Para a estimação dessas equações, este estudo propõe a utilização de Dados em Painel, conforme defendido inicialmente por Vermeulen (1994). No Brasil, as empresas divulgam informações contábeis apenas trimestralmente, informações essas necessárias ao cálculo do fluxo de caixa. 0 procedimento proposto visa aumentar o volume de dados para a estimação, principal mente ao analista externo à empresa, al ém de permitir a consideração de características que a diferenciam das demais quando da estimação de seu fluxo de caixa.

\section{Tratamento dos fatores de risco próprios e estimação do comportamento médio esperado dos fatores de risco macroeconômicos}

Realizada a estimação via Dados em Painel e identificados os fatores de risco relevantes entre os candida-

Quadro 1 - Modelo contábil de fluxo de caixa livre.

\section{LUCRO LÍQUIDO DO EXERCÍCIO}

( \pm ) Fatores que não afetam caixa (despesas de depreciação, resultado de equivalência patrimonial, outras receitas e despesas não caixa)

(+) Variação em Duplicatas a Receber

(t) Variação em Estoques

(t) Variação em Fornecedores

(+) Variação em outros itens circulantes

(=) Fluxo de caixa gerado (consumido) pelas operações

(-) Compra de ativo permanente

(t) Venda de ativo permanente

(=) Fluxo de caixa após as atividades de investimento

(+) Variações no nível de endividamento e/ ou no capital social da empresa

(-) Pagamento de dividendos e/ ou juros sobre o capital próprio

(=) Fluxo de caixa após as atividades de financiamento

Fonte: Manual de Contabilidade das S.A. (2000). 
tos pré-selecionados, o próximo passo do modelo é estimar o comportamento médio esperado desses fatores ao longo do tempo.

Ressal te-se que, por sua característica mais estável, os fatores de risco próprios da empresa não têm seu comportamento futuro projetado neste modelo. Espera-se que, numa situação real, a empresa tenha ciência dos valores futuros a serem assumidos por tais fatores via planejamento periódico: market share pretendido, nível de endividamento, contratação ou amortização de dívidas em moeda nacional ou estrangeira, novos investimentos, venda de ativos, novos aportes de capital e transferência de recursos para os acionistas. Na modelagem, os valores assumidos pelos fatores de risco próprios são mantidos constantes e iguais ao último dado disponível. Nada impede, entretanto, que, no caso de uma pesquisa interna, realizada com informações privadas, tais valores esperados sejam fornecidos pela empresa, de acordo com sua estratégia de atuação.

Portanto, o foco neste passo está na estimação dos valores futuros (média condicional) dos fatores de risco macroeconômicos. Tal como sugere o documento CorporateM etrics (RiskM etrics Group, 1999), neste modelo a estimação é feita via Vetores Auto-Regressivos (VARM), com a inclusão do mecanismo de correção de erros (ECM) sempre que necessário. Destaquese que o objetivo da modelagem empregada não é acertar pontualmente os valores para as variáveis macroeconômicas relevantes, mas apenas acessar sua provável distribuição futura e utilizar tal informação para a construção da distribuição futura da variável de interesse (fluxo de caixa livre, ou FCF). Em linha com tal objetivo, esse modelo sugere a estimação das variáveis macroeconômicas conjuntamente, via Vetores AutoRegressivos Irrestritos (Sims, 1980, apud Enders, 1995).

Para estimar tais vetores, as variáveis devem manter relações teóricas entre si, sendo cada uma delas passível de influenciar seu próprio comportamento no futuro e também o comportamento das demais. Como já ressaltado, o objetivo primordial dessa estimação é encontrar relações importantes (elongas) entre as variáveis e não fazer estimativas pontuais.

Para a anál ise do mel hor model o estimado, de acordo com tal metodologia, é recomendável não se ater à significância dos parâmetros, uma vez que os regressores geralmente são altamente colineares. Portanto, testes $t$ convencionais não são recomendados para definir o melhor modelo. Nessa estimação, 0 melhor modelo é aquele que minimiza a soma dos erros ao quadrado, sendo também parcimonioso em re- lação ao número de regressores. Portanto, recomenda-se a adoção de dois critérios que levem em conta a soma dos erros ao quadrado, penalizando o modelo com muitos regressores: O AIC (Akaike Information Criteria) e o SBC (Schwarz's Bayesian Criterion). O melhor modelo é o que apresenta os menores valores de AIC ou SBC entre aqueles considerados.

Por fim, para proceder à estimação via VARM, devem ser previamente empreendidos alguns testes que verifiquem se as séries mantêm ou não relações teóricas entre si (Testes de Granger Causalidade), e que identifiquem a existência de não estacionariedade (Testes de Raiz Unitária), com consideração de quebras estruturais, e a possibilidade de elas serem co-integradas ao nível.

\section{Simulação de cenários para os fatores de risco macroeconômicos}

Escolhido o modelo VARM para a previsão dos fatores de risco macroeconômicos, sua aplicação permite a previsão conjunta dos valores médios de tais fatores $j$ passos à frente. Portanto, a partir de tal modelo, temse um único valor previsto para cada fator de risco (equival ente à sua média condicional projetada). Para construir uma distribuição para cada fator de risco, a ser inserida nas equações que relacionam tais fatores ao fluxo de caixa - gerando, portanto, uma distribuição de fluxo de caixa - , torna-se necessário gerar n potenciais choques sobre esses fatores.

A forma usual mente empregada para gerar tais choques é via Simulação de M onte Carlo. N essa simulação há o sorteio al eatório de perturbações - números al eatórios extraídos de uma Distribuição $\mathrm{N}$ ormal $(0,1)$ - que se somam à média do fator, prevista via modelo VARM. Considerando-se que tais perturbações sorteadas precisam manter a estrutura de variânciacovariância histórica observada entre os fatores, os erros gerados pelo modelo VARM (diferença entre os val ores históricos observados e sua média prevista) são utilizados para construção da matriz de variânciacovariância dos fatores $(\Sigma)$, que filtrará, via fatoração de Cholesky, os números al eatórios sorteados (choques).

\section{Determinação da estatística de interesse}

Estimados, via VARM, os valores mais prováveis (média) para cada fator de risco macroeconômico, em cada data futura de interesse $j$, e estressad os tais val ores pela consideração de $n$ possíveis choques aleatórios, os n potenciais valores futuros de cada fator de risco são inseridos na equação que relaciona tais fatores ao fluxo de caixa livre (FCF) da empresa. Torna-se impor- 
tante destacar, conforme já mencionado, que, nesse passo, os fatores de risco próprio são mantidos constantes ou fornecidos pela empresa.

Encontra-se, dessa forma, uma distribuição empírica para o fluxo de caixa livre da empresa, em cada data futura de interesse j. A partir dessa distribuição, é possível estimar a área sob $\mathrm{FCF}<0$, em cada data futura de interesse. Tal área é considerada neste estudo como igual à probabilidade de a empresa, na data futuraj, não dispor de recursos para honrar seus compromissos. Explorados todos os passos, o modelo pode ser resumido pelo esquema exposto na Figura 1.

\section{MODELO EMPÍRICO PARA CASHFONAATFISK}

Exposta a metodologia teórica proposta para a estimação do fluxo de caixa em risco, este tópico destina-se à aplicação empírica do modelo teórico desenvolvido às empresas pertencentes ao setor de distribuição de energia elétrica brasileiro.

\section{Amostra de trabalho e dados coletados}

Para o desenvolvimento do modelo foram coletados dados das empresas brasileiras distribuidoras de ener- gia elétrica que publicaram seus demonstrativos contábeis, em base regular, por meio do Sistema de Divulgação Externa (DivExt) da CVM. A amostra final contou com 25 empresas, apresentadas na Tabela 1.

A partir da análise dos demonstrativos contábeis dessas distribuidoras, extraíram-se, em base trimestral, desde quando disponível - máximo de 34 trimestres, considerando-se dados obtidos desde 0 10T/95 até o 2ำ T/03 - , as informações constantes do Quadro 2.

Algumas das informações constantes no Quadro 2 foram utilizadas na construção da variável dependente do modelo (fluxo de caixa livre), conforme detaIhado no próximo item. As demais foram testadas como variáveis independentes; portanto, passíveis de determinar oscilações nos fluxos de caixa livres das empresas.

\section{Determinação do fluxo de caixa livre de cada empresa $\mathbf{i}$, na data $\mathbf{t}$}

Antes de proceder à esti mação dos fatores determinantes de oscilações nos fluxos de caixa livres das empresas, houve necessidade de escolher uma proxy para tal fluxo. De forma a viabilizar a obtenção do fluxo de cai xa livre contábil para todas as 25 empresas da amostra, nos 34 trimestres considerados, optou-se por em-

Figura 1 - Modelo teórico esquematizado.

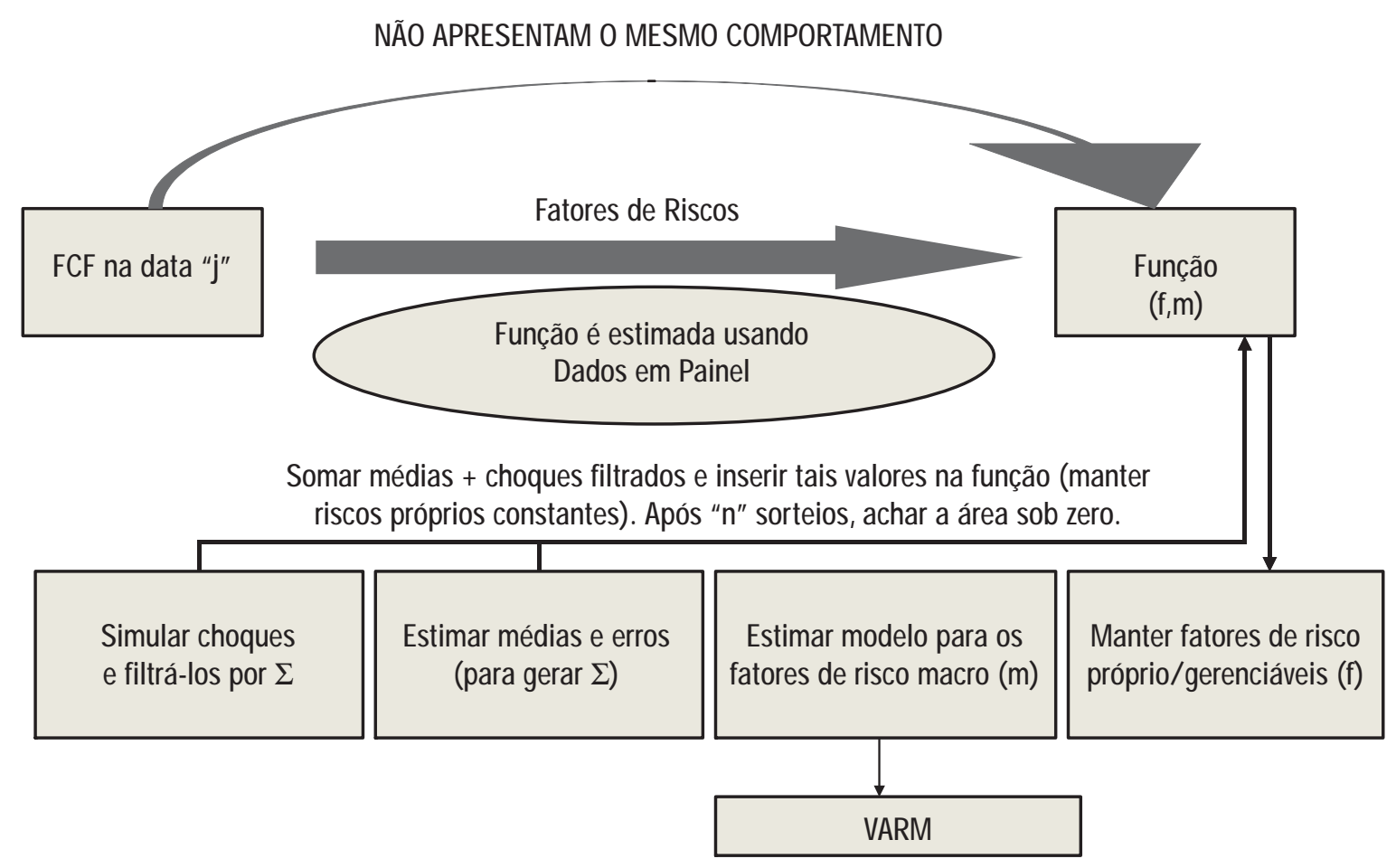


preender algumas simplificações do modelo contábil de fluxo de caixa livre, necessárias ao analista externo à empresa, a saber:

- utilizou-seo lucro operacional do exercício (receita bruta menos deduções, menos custo do produto vendido, menos despesas operacionais, menos despesas financeiras líquidas), acrescido da despesa de depreciação (quando esta tivesse sido previamente descontada como despesa operacional). Com isso, eliminaram-se contas não caixa, tais como resultado de equivalência patrimonial;

- consideraram-se os estoques como iguais a zero e prazos médios de recebimento de clientes iguais aos prazos médios de pagamento aos fornecedores. Com isso, foram eliminadas as necessidades de capital de giro das empresas;

- utilizaram-se as variações na conta Ativo Permanenteobtidas pela DOAR e rateadas trimestralmente de acordo com o percentual de despesas operacionais do tri- mestre em relação às despesas operacionais anuais como proxy para gastos de capital (Capital Expenditures ou CapEx);

- empregaram-se as variações na conta Empréstimos e Financiamentos de Curto e Longo prazo - obtidas via Balanço Patrimonial -, assumindo-as como alterações líquidas no financiamento (contratação de novas dívidas, mais encargos financeiros apropriados por competência mas não desembolsados, menos amortizações).

A variável assim criada, para cada empresa i, em cada data t, foi denominada fluxo de caixa livre (FCF). N este estudo, foi previsto o fluxo de caixa livre (FCF) de empresas distribuidoras de energia 2 períodos à frente $(\mathrm{j}=2)$, em vértices trimestrais. Para tanto, trabalhouse com os dados disponíveis até o 4 $\mathrm{T} / 02$, reservando-se os dados do $1^{\circ}$ e $2^{\circ}$ trimestres de 2003 para procedimentos de backtesting.

Tabela 1 - Empresas na amostra.

\section{NOME DA EMPRESA}

1. Cia. Energética de Minas Gerais

2. Cia. Paranaense de Energia

3. Caiuá Serviços de Eletricidade S.A.

4. AES Sul Distribuidora Gaúcha de Energia S.A

5. Cia. Eletricidade da Bahia

6. Eletropaulo Metropolitana (AES Eletropaulo)

7. Espírito Santo Centrais Elétricas S.A.

8. Light Serviços de Eletricidade S.A.

9. Cia. Energética de Brasília S.A.

10. Cia. Energética de Pernambuco

11. Cia. Energética de Goiás

12. Cia. Energética do Maranhão

13. Elektro Eletricidade e Serviços S.A.

14. Rio Grande Energia S.A.

15. Energisa S.A.

16. Empresa Energética do Mato Grosso do Sul S.A.

17. Cia. Força e Luz Cataguases-Leopoldina

18. Bandeirante Energia S.A.

19. Centrais Elétricas de Santa Catarina S.A.

20. Centrais Elétricas do Pará S.A.

21. Centrais Elétricas Mato-Grossenses S.A.

22. Cia. Eletricidade do Rio de Janeiro

23. Cia. Energética do Ceará

24. Cia. Energética do Rio Grande do Norte

25. Cia. Estadual de Energia Elétrica

\section{SIGLA UTILIZADA}

MG

PR

REDE

AES

BA

$S P$

ES

LIG

CEB

PE

GO

MA

ELK

RGE

GISA

MS

CTLEO

EBE

$S C$

PA

MT

RJ

CE

RN

CEE
INFORMAÇÕES DESDE

$10 \mathrm{~T} / 95$

10 T/ 95

10 T/ 95

3ㅇ $\mathrm{T} / 97$

1ㅇ T/ 95

10 T/ 96

10 T/ 95

$10 \mathrm{~T} / 95$

$10 \mathrm{~T} / 98$

10 T/ 95

10 T/ 95

$10 \mathrm{~T} / 96$

20 T/98

3ㅇ $\mathrm{T} / 97$

1ㅇ $\mathrm{T} / 98$

$10 \mathrm{~T} / 95$

10 T/ 95

$10 \mathrm{~T} / 98$

$10 \mathrm{~T} / 95$

10 $\mathrm{T} / 98$

$10 \mathrm{~T} / 96$

$10 \mathrm{~T} / 95$

$10 \mathrm{~T} / 95$

$10 \mathrm{~T} / 98$

1ㅇ T/ 95 
Em relação aos vértices do fluxo (datas de interesse), destaca-se que a existência de dados em base trimestral, menor desagregação possível de informações públicas, foi responsável pela escolha dos vértices trimestrais de pagamento nessa aplicação. Portanto, os vértices do fluxo foram sempre os meses de março, julho, setembro e dezembro de cada ano.

Quadro 2 - Variáveis de trabalho.

I. Variáveis retiradas da Demonstração do Resultado do Exercício (DRE):

a) receita bruta trimestral (em $R \$)$;

b) deduções à receita bruta trimestral (em $R \$)$;

c) custo dos produtos vendidos (em $R \$$ );

d) despesas operacionais (em $R \$$ );

e) despesas financeiras líquidas (em R\$);

II. Variáveis retiradas da Demonstração da Origem e Aplicação dos Recursos (DOAR)

f) depreciação (em $R \$$ );

g) variação do Ativo Permanente: Investimentos, Imobilizado e Diferido (em R\$).

III. Variáveis retiradas do Relatório Anual ou Comentário de Desempenho do Trimestre

h) mercado atendido (em GWh);

i) número de consumidores atendidos;

j) reajuste anual de fornecimento (\% e data de aplicação);

k) indicador de duração de interrupção no atendimento (DEC);

l) indicador de freqüência de interrupção no atendimento (FEC);

m) investimentos realizados no período (em $R \$$ )

n) estoque de dívida em moeda estrangeira (em $R \$)$;

o) estoque de dívida em moeda nacional (em R\$);

IV. Variáveis retiradas de fontes diversas

p) Tarifa Média de Fornecimento - Brasil (em R\$)

Fonte: Eletrobrás, desde fev/ 90

q) PIB (dessazonalizado, a preços de mercado, base $1990=100$ )

Fonte: Banco Central do Brasil, desde mar/ 91

r) Indicador do Nível de Atividade - INA (base $1991=100$ )

Fonte: FIESP, desde jan/ 86

s) Indicador da Produção Industrial (base $1991=100$ )

Fonte: Instituto Brasileiro de Geografia e Estatística, desde jan/ 80

t) M1 (média dos dias úteis do mês - em $R \$$ )

Fonte: Banco Central do Brasil, desde jan/ 80

u) IPCA (\% mensal)

Fonte: Instituto Brasileiro de Geografia e Estatística, desde jan/ 80

v) IGP-M (\% mensal)

Fonte: Economática, desde jan/ 90

w) $T$ J LP (\% anual)

Fonte: Economática, desde dez/ 94

x) Selic (\% anual)

Fonte: Economática, desde jan/ 90

y) PTAX (R\$/US\$)

Fonte: Economática, desde jan/ 90

z) C-BOND (preço de fechamento - em R\$)

Fonte: Bloomberg, desde dez/ 94

aa) Consume Price Index - CPI/ USA (\% mensal)

Fonte: Bloomberg, desde jan/ 84

Nota: As informações contábeis acima coletadas foram referentes às empresas controladoras, expressas em moeda do período, sem nenhuma indexação ou correção monetánia. 


\section{Escolha das variáveis independentes}

A escolha das candidatas a variáveis independentes foi limitada pela disponibilidade de dados existentes e pela necessidade de se chegar a resultados parcimoniosos, que permitissem a estimação de vetores auto-regressivos. Assim, a opção foi considerar as seguintes variáveis como explicativas:

- despesas com compra de energia (30\% da receita bruta da empresa, em R\$);

- mercado atendido pela empresa (em GWh);

- número de consumidores atendidos pela empresa;

- reajuste anual de fornecimento concedido à empresa (\% anual igualmente rateado nos trimestres);

- indicador de duração de interrupção no atendimento da empresa (DEC);

- indicador de freqüência de interrupção no atendimento da empresa (FEC);

- estoque de dívida em moeda estrangeira mantido pela empresa (em R\$);

- estoque de dívida em moeda nacional mantido pela empresa (em R\$);

- Tarifa M édia de Fornecimento - Brasil (\% trimestral);

- PIB, INA e Produção Industrial (\% trimestral);

- IPCA, IGPM e M 1 (\% trimestral);

- TJLP e Selic (\% trimestral);

- PTax (\% trimestral);

- C-Bond e CPI/USA (\% trimestral).

As variáveis PIB (Produto Interno Bruto), INA (Indicador do Nível de Atividade) e Produção Industrial foram incluídas como proxies para nível de atividade no setor industrial e comercial; IPCA (Índice Geral de Preços - Amplo), IGPM (Índice Geral de Preços Mercado) e M 1 (papel-moeda em circulação) como proxies para inflação, sendo o IGPM o indexador das receitas e de al gumas das despesas das concessionárias; TJLP (Taxa de Juros de Longo Prazo) e Selic (taxa básica da economia brasileira) como proxies para taxas de juros, que limitam o consumo das famílias, ao mesmo tempo em que indexam uma parte do endividamento das empresas; PTax para câmbio, já que grande parte da dívida do setor é em moeda estrangeira, e CBond e CPI/USA (Consumer Price Index) como proxies para atração de capital estrangeiro.

É importante ressaltar que a escolha, a priori, do conjunto de potenciais variáveis explicativas não obedeceu a nenhum critério estatístico, mas, diferentemente, foi empreendida tomando-se por base apenas o estudo do setor. Obviamente, ao escolher um conjunto tão restrito de variáveis, deixou-se de fora um vasto campo de pesquisa. Entretanto, o método selecionado para previsão dos fatores de risco macroeconômicos (vetores auto-regressivos) exige parcimônia do pesquisador, uma vez que, para um conjunto de " $x$ " variáveis, há que se estimarem conjuntamente " $\mathrm{x}$ " parâmetros para cada defasagem temporal considerada. $\mathrm{N}$ ada impede que, em estudos posteriores, outras variáveis explicativas sejam consideradas.

\section{Identificação dos fatores de risco relevantes: estimação em painel}

Para a identificação dos fatores de risco do FCF, adotou-se o seguinte procedimento: (a) estimaram-se, via painel, os fatores de risco de cada componente do FCF separadamente, quais sejam: receita bruta, despesas operacionais e despesas financeiras líquidas; (b) empregou-se, como estimativa das deduções (como impostos e abatimentos), a média histórica de cada empresa, dado que tal conta apresentou pouquíssima variabilidade; (c) considerou-se CapEx e variação do endividamento como contas gerenciáveis, ou seja, passíveis de serem alterados pela empresa de acordo com as condições do ambiente macroeconômico. Com isso, decidiu-se não estimar fatores de risco para tais componentes do fluxo de caixa e utilizar o último valor disponível como a melhor estimativa para o período. Ao final da estimação, as contas componentes do fluxo de caixa livre foram novamente somadas, de modo a restaurar a variável de interesse (FCF).

Considerando-se que o estudo utilizou unicamente informações públicas - obtidas de fora da empresa - e que as empresas apenas divulgam informações contábeis em base trimestral, a opção pela estimação em painel aumentou consideravelmente o volume de informações disponíveis. Caso a opção fosse por trabalhar com uma única empresa, observada desde 1995, haveria 32 trimestres para estimação e 2 para backtesting. Ao trabal har com 25 empresas observadas ao longo do tempo, chegou-se a 690 dados para estimação e 50 para backtesting. A seguir, apresentam-se os resultados da estimação via dados em painel, realizada no software STATA 8.0. ${ }^{1}$

Os resultados para a variável "receita bruta" estão detal hados na Tabela 2. Ressalte-se que houve necessidade de se incluir uma dummy para o período posterior ao 4ํT/01 (variável DRTE2), data de concessão do Reajuste Tarifário Extraordinário, que compensava as perdas do racionamento.

É interessante notar que todas as variáveis independentes apresentaram os sinais esperados para os coefi- 
cientes (dummy DRTE2, Mercado em GWh, IGPM e Selic), à exceção da variável "produção industrial" (Prodlnd), negativamente correlacionada às receitas apuradas. Prováveis explicações para tal relação são as tarifas mais baixas cobradas de consumidores industriais e a opção, cada vez mais recorrente, das indústrias pela autoprodução em períodos de pico da atividade econômica.

A Tabela 2 também apresenta os resultados para a variável "despesas operacionais", que engloba tanto custos quanto despesas operacionais propriamente ditas. Para a determinação dessa variável foi incluída uma dummy em jan/99 (variável DCâmbio), data da desvalorização cambial, já que parte da energia comprada pelas distribuidoras das regiões Sul e Sudeste vem de I taipu, cujas tarifas são indexadas ao dólar. A dummy DRTE difere da DRTE2 por considerar o efeito da revisão tarifária apenas sobre o 40 T/01.

Por fim, a mesma tabel a apresenta os resultados para a última variável estimada: "Despesas Financeiras Líquidas". Na determinação dessa variável foi incluída uma dummy em jan/99 (data da desvalorização cambial) .
Foram significantes as variáveis: dummy (DCâmbio), estoque de dívida estrangeira (DivEst), estoque de dívida nacional (DivN ac), PTax e CBond.

\section{Estimação do comportamento médio esperado dos fatores de risco macroeconômicos: vetores auto-regressivos irrestritos}

Realizada a estimação em painel e identificados os fatores de riscos, procedeu-se à estimação da média condicional dos fatores de risco macroeconômicos via vetores auto-regressivos. Como visto na estimação anterior, foram identificadas as seguintes variáveis explicativas (fatores de risco):

- mercado atendido pela concessionária (em GWh) Mercado

- estoque de dívida em moeda nacional (em R\$) - DivNac

- estoque de dívida em moeda estrangeira (em R\$) - DivEst

- produção industrial (\% trimestral) - Prodlnd

- IGPM (\% trimestral) - IGPM

- Selic (\% trimestral) - Selic

- PTax (\% trimestral) - PTax

- CBond (\% trimestral) - CBond

Tabela 2 - Estimação dos fatores de risco de "receita bruta", "despesas operacionais" e "despesas financeiras líquidas" via Painel/ Efeitos Fixos

\begin{tabular}{|l|c|c|c|c|}
\hline VARIÁVEL ESTIMADA & VARIÁVEIS INDEPENDENTES & COEFICIENTES & ESTATISTICA t & p-value \\
\hline Receita Bruta & drte2 & $186.308,40$ & 11,30 & 0,000 \\
\hline (rec) & mercado & 0,03 & 3,71 & 0,000 \\
& prodind & $-2.109,88$ & $-3,72$ & 0,000 \\
& igpm & $4.898,77$ & 2,66 & 0,008 \\
& selic & $-29.761,00$ & $-11,91$ & 0,000 \\
& cons & $413.917,5$ & 16,82 & 0,000 \\
\hline Despesas & drte & $167.686,30$ & 6,55 & 0,000 \\
\hline Operacionais & dcambio & $60.828,77$ & 3,81 & 0,000 \\
(desptot) & mercado & 0,02 & 0,001 \\
& prodind & $-1.516,76$ & $-3,33$ & 0,002 \\
& igpm & $8.376,15$ & 5,16 & 0,000 \\
& selic & $-21.341,1$ & $-10,74$ & 0,000 \\
& cons & $271.991,4$ & 13,67 & 0,000 \\
\hline Despesas & dcambio & $92,838,19$ & 6,26 & 0,000 \\
Financeiras Líquidas & divest & 0,07 & 13,35 & 0,000 \\
(despfin) & divnac & 0,03 & 2,60 & 0,010 \\
& ptax & $1.562,29$ & 4,35 & 0,000 \\
& cbond & $-1.160,70$ & $-4,24$ & 0,000 \\
& cons & $-23.952,44$ & $-4,34$ & 0,000 \\
\hline
\end{tabular}


As três primeiras variáveis foram consideradas como gerenciáveis, ou seja, passíveis de serem programadas pela empresa. Portanto, os esforços nessa etapa se concentraram nos possíveis choques sofridos pelas variáveis ProdInd, IGPM, Selic, PTax e CBond.

Ressalte-se que o objetivo precípuo aqui não era prever pontualmente os valores médios para as cinco variáveis macroeconômicas citadas, mas apenas acessar sua provável distribuição futura eutilizar tal informação para construção da distribuição futura da variável de interesse (FCF). Em linha com tal objetivo, empreendeu-se a estimação das cinco variáveis macroeconômicas conjuntamente, via Vetores Auto-Regressivos I rrestritos.

Antes de proceder à estimação do VARM Irrestrito anteriormente mencionado, foi necessário empreender testes de raiz unitária a cada uma das séries consideradas. Possuindo as séries o mesmo número de raízes unitárias, seria preciso checar a possibilidade de serem co-integradas. Observando-se os gráficos das séries consideradas, constatou-se uma quebra estrutural em jul/94, mês de implementação do Plano Real, para as séries Selic, IGPM e PTax. Adicionalmente, a série PTax sofreria outra mudança estrutural em jan/ 99 (mês de liberação do câmbio para o regime flutuante). A série Prodlnd mostrava comportamento constante e a série CBond (iniciada em dez/94) também.

Portanto, procedeu-se ao teste de raiz unitária com quebra estrutural de Perron para as três primeiras séries (Selic, IGPM e PTax) e ao teste de raiz unitária de Phillips-Perron para as duas últimas (Prodlnd e CBond). Como os testes de raiz unitária não revelaram a existência de raízes (após a consideração das quebras estruturais), consideraram-se as séries como estacionárias, el iminando-se a necessidade de diferenciação e a possibilidade de haver co-integração entre elas. Portanto, o VARM Irrestrito pode ser estimado sem mecanismo de correção de erros (ECM).

Logo após, com o objetivo de verificar se as séries consideradas na estimação do VARM eram, de fato, correlacionadas entre si, procedeu-se aos testes de Granger Causalidade. 0 objetivo desse teste é revelar se determinada variável defasada explica parte da variância de outra. N este estudo, realizaram-se testes de Granger Causalidade para lags $=3,2$ e 1 .

Os testes de Granger Causalidade (com 10\% de significância, para número de lags $=3$ ), revelaram que: Selic causa Prodlnd; IGPM causa Selic, ProdInd e CBond; PTax causa CBond e ProdInd; e CBond causa IG PM;

Constatadas as causalidades entre as variáveis macroeconômicas, procedeu-se à estimação do VARM I rrestrito. Foram avaliados modelos com 3, 2 e 1 lag. A eficácia de cada um dos modelos está representada na Tabela 3.

De acordo com o critério de minimização do AIC, 0 modelo escolhido foi o VARM com 3 lags. A pesar de esse modelo apresentar um SBC superior, com 3 defasagens há relações de causalidade entre as variáveis Selic, IGPM , PTax e a variável Prodınd, o que não ocorre quando o model o considera apenas um ou dois lags.

\section{Equações do modelo}

Esquematicamente, o model o final pode ser visual izado na Figura 2.

\section{RESULTADOS}

Após a estimação das médias para os fatores de risco 1 e 2 passos à frente $(j=2)$, procedeu-se à construção da matriz de variância-covariância $(\Sigma)$ desses fatores a partir dos resíduos gerados na estimação. Tal matriz foi decomposta via Fatoração de Cholesky, servindo sua matriz triangular inferior de filtro para mil choques aleatórios extraídos de uma distribuição normal $(0,1)$.

Para procedimentos de backtesting, esses choques, agora filtrados, foram somados aos val ores médios dos fatores de riscos, em cada trimestre de interesse ( $1^{\circ} \mathrm{e}$ 20 trimestres de 2003), e inseridos nas relações econométricas que determinavam a "receita bruta", "despesas operacionais" e "despesas financeiras líquidas", gerando mil potenciais valores para cada conta, em cada data de interesse.

Tabela 3 - Comparação de VARM Irrestritos.

\begin{tabular}{l|l|l}
\multicolumn{1}{|c|}{ VARM 3 LAGS } & \multicolumn{1}{c}{ VARM 2 LAGS } & \multicolumn{1}{c}{ VARM 1 LAG } \\
$\mathrm{R}^{2}=0,81$ & $\mathrm{R}^{2}=0,82$ & $\mathrm{R}^{2}=0,82$ \\
$\mathrm{R}^{2}$ Ajustado $=0,63$ & $\mathrm{R}^{2}$ Ajustado $=0,74$ & $\mathrm{R}^{2}$ Ajustado $=0,79$ \\
$\mathrm{AIC}=2,97$ & $\mathrm{AIC}=2,98$ & $\mathrm{AIC}=3,07$ \\
$\mathrm{SBC}=3,68$ & $\mathrm{SBC}=3,45$ & $\mathrm{SBC}=3,30$
\end{tabular}


Conforme já mencionado, para montar o FCF, 0 percentual de "deduções" no $1^{\circ}$ e 20 trimestres de 2003 foi considerado como igual à média histórica de cada empresa e aplicado sobre a receita bruta estimada; as variáveis C apEx e variação da dívida foram consideradas como iguais às verificadas no último trimestre disponível. A puradas todas contas, foram somadas para restaurar a variável FCF.

Ao final do processo, obteve-se um histograma para cada empresa da amostra, em cada trimestre de interesse ( 10 e 20 trimestres de 2003), e estimou-se a probabilidade de a empresa apresentar um FCF negativo (percentil relacionado à estatística de ordem zero). A Figura 3 apresenta os valores reais do FCF, os valores previstos a partir das médias dos fatores de risco macroeconômicos e um intervalo de confiança para as previsões (média \pm 2 desvios padrão).

A Tabela 4 apresenta os resumos dos fluxos de cai- xa e a probabilidade de o FCF de cada empresa, em cada trimestre, ser inferior a zero. Em destaque estão as empresas e trimestres nos quais o intervalo de confiança apurado continha os valores reais dos fluxos.

\section{DISCUSSÃO E CONCLUSÕES}

Froot, Scharfstein e Stein (1994) enfatizam que um programa de gerenciamento de riscos de mercado deve ter como único objetivo assegurar um planejamento acurado dos recursos necessários para os investimentos da empresa e a manutenção de sua atividade produtiva. Como subproduto relevante desse gerenciamento, os autores também destacam a capacidade que as empresas adquirem de avaliar quais riscos devem ser evitados, hedgeados ou simplesmente enfrentados por serem parte integrante de seu negócio.

Figura 2 - Equações do modelo.

\section{Identificação dos Fatores de Risco Relevantes}

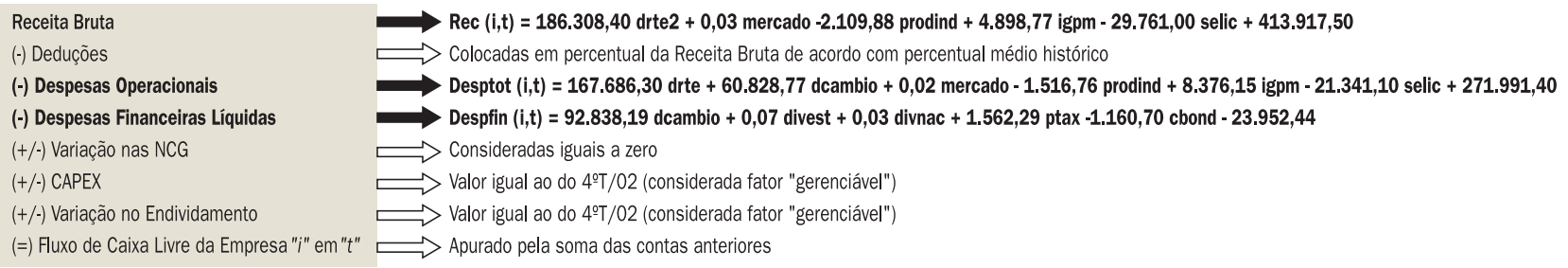

\section{$\longrightarrow$ Estimação das Equações via Dados em Painel}

$\Longrightarrow$ Apuração conforme descrito

\section{Estimação do Comportamento Médio Esperado dos Fatores de Risco Identificados}

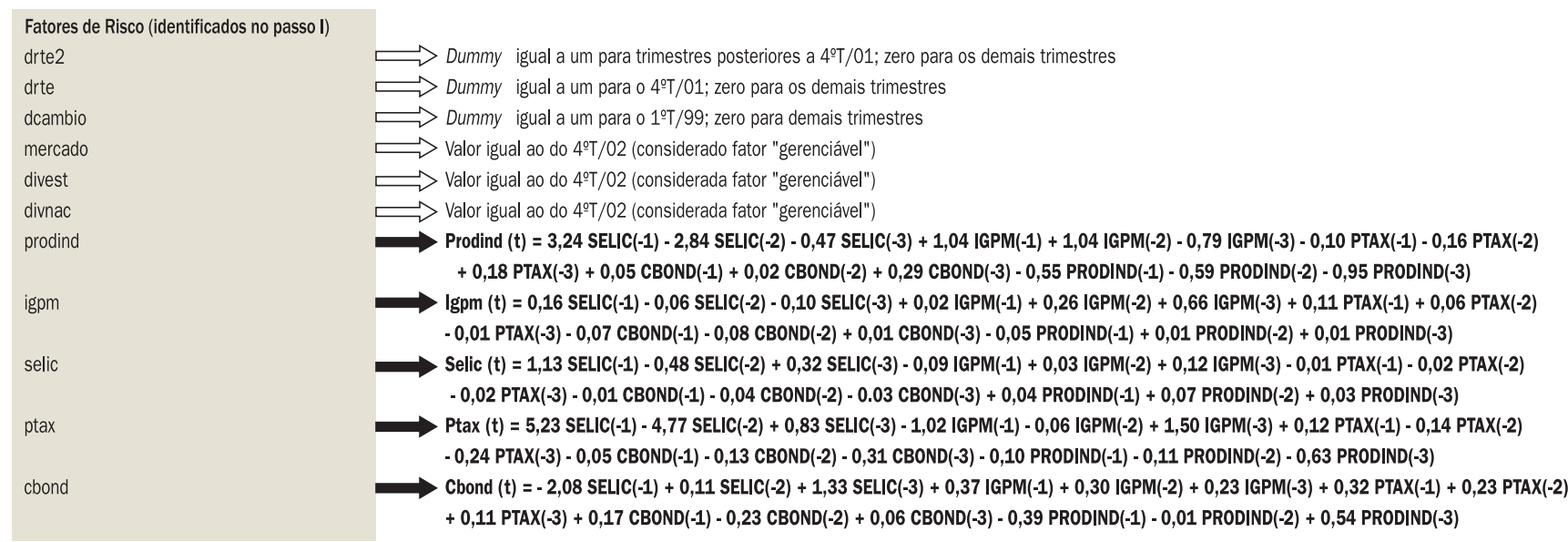

$\longrightarrow$ Estimação das Equações via VARM

$\longrightarrow$ Apuração conforme descrito 
Indo um pouco mais adiante, o guia para gerenciamento de riscos do JP M organ eArthur Andersen (1997) enfatiza que, uma vez que as exposições a risco da empresa estejam identificadas e quantificadas, o próximo passo é trabalhar o perfil de risco da empresa. Essa remodelação passa, por exemplo, por alterações no perfil da dívida, compati bilização dos prazos e dos termos de pagamento e de recebimento, e adequação do cronograma de investimentos aos recursos disponíveis.

Visando servir de ferramenta a tal gerenciamento, 0 modelo aqui apresentado e aplicado às empresas distribuidoras de energia elétrica, a despeito de ter sido empiricamente construído de fora da empresa e de prescindir de informações gerenciais preciosas - especialmente em relação aos valores futuros dos fatores de risco próprios -, demonstra ser útil aos gestores para avaliar o comportamento futuro de seus fatores de risco macroeconômicos e, principalmente, 0 reflexo de tal comportamento sobre os fluxos de caixa projetados. Munidos de tal metodologia, o budget plan elaborado pelos gestores evolui de um cenário único para uma distribuição de fluxos futuros, obtida pela consideração de movimentos diversos nas premissas do orçamento elaborado. Com isso se ganha um instrumento mais sofisticado de anál ise de sensibilidade. A sofisticação advém da consistência da previsão da média condicional dos fatores de risco macroeconômicos e dos choques simulados, ao contrário da análise de sensibilidade convencional, que insere choques não correlacionados em diversas variáveis rel evantes - quando, na realidade, tais choques são totalmente correla- cionados. Por meio do modelo proposto é possível inserir no budget plan elaborado choques que mantenham a estrutura de correlação histórica entre as variáveis e através do tempo.

Entretanto, é importante reconhecer as limitações de tal modelo. De maneira geral, ressalta-se o fato de o presente modelo ser apenas uma primeira abordagem de uma pesquisa mais ampla. Dessa forma, serão necessários mais testes, com outras especificações econométricas, além da aplicação em outros setores, até que se chegue a um consenso sobre a especificação que considere o maior número possível de fatores de risco, a melhor forma de previsão desses fatores e de sua relação com as contas do fluxo de caixa. Com 0 objetivo de poder responder a perguntas do tipo "0 modelo proposto tem mais poder de previsão que um modelo mais simples?", há que se dar prosseguimento ao trabalho, considerando outras técnicas de medição do fluxo de caixa em risco, como a Simulação Histórica e Simulação de M onte Carlo com Equações Estruturais, em outros setores da economia.

Como limitações inerentes à metodologia empregada nesse trabalho, destacam-se tanto a escolha a priori do escopo de candidatas a variáveis independentes como a parcimônia necessária na decisão de quais fatores de risco macroeconômico considerar na modelagem econométrica. A dicional mente, não há garantias de que será sempre possível estimar o comportamento futuro dos fatores de risco via modelos auto-regressivos. Especialmente quando o horizonte de previsão for de prazo mais longo, há restrições à eficácia de tais modelos.

Figura 3 - Fluxos de caixa reais e previstos.

Fluxos de Caixa - 1 e 2 Trimestres de 2003

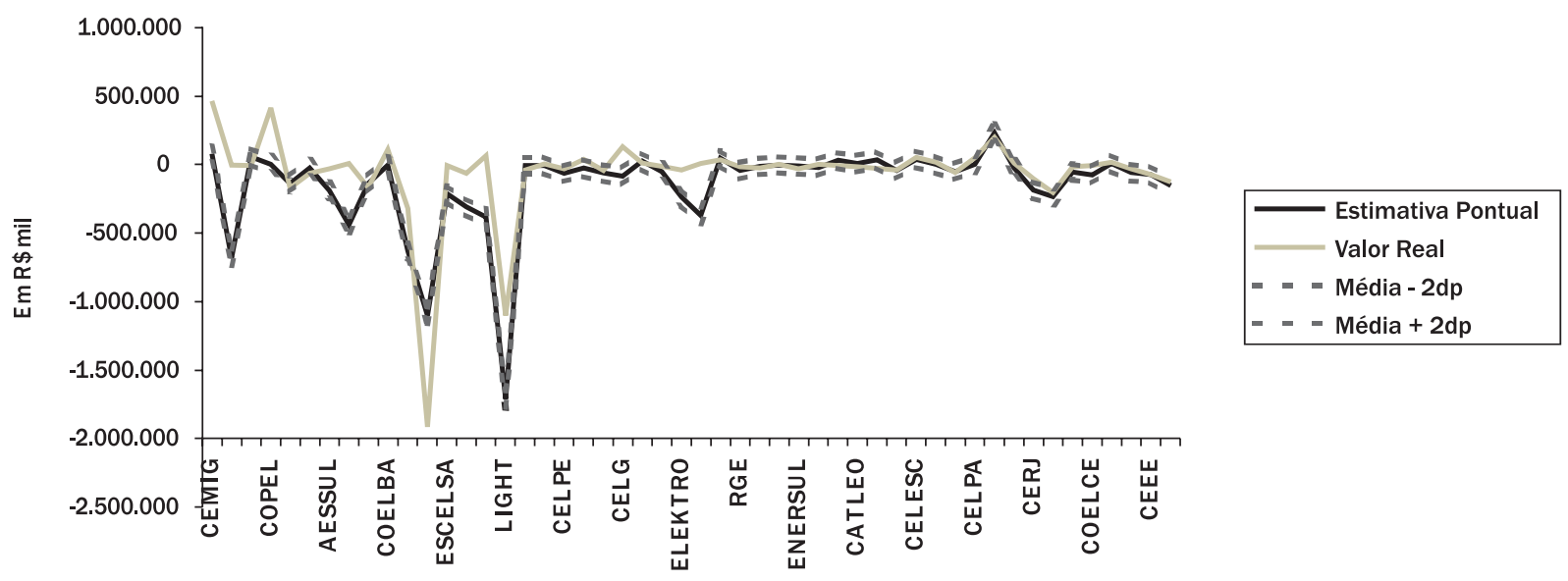




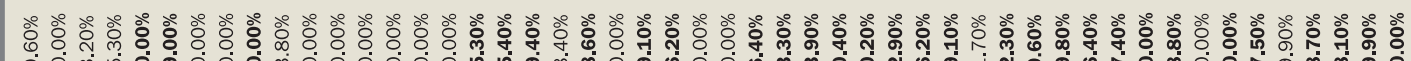

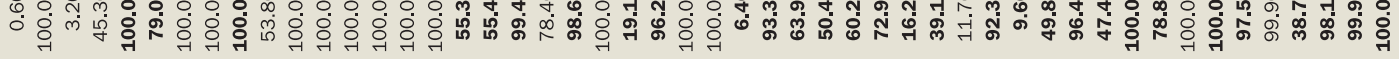

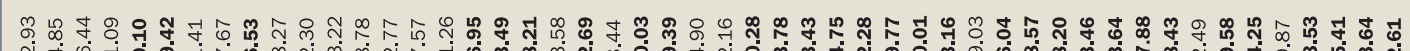

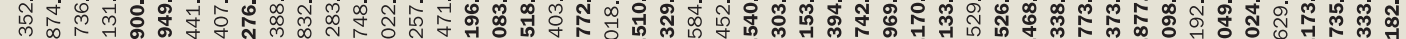

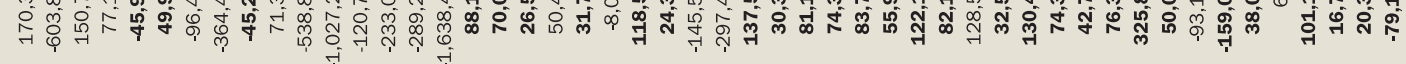

ల क o

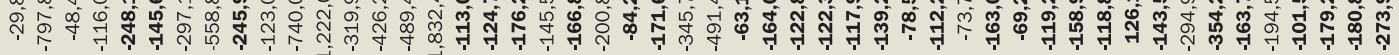

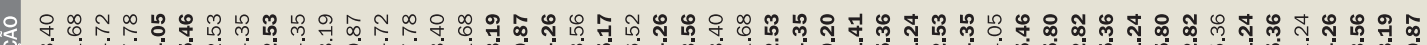

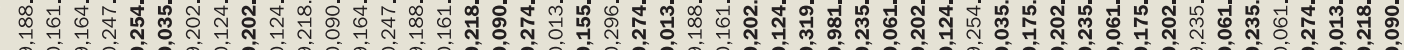
ลำ

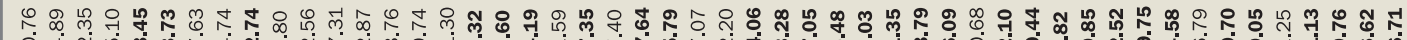
ठ্户

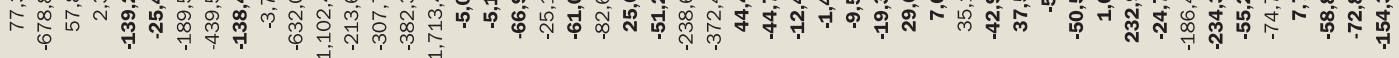

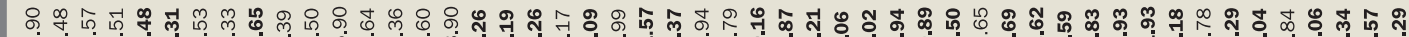
ơ 人

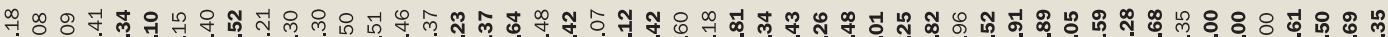

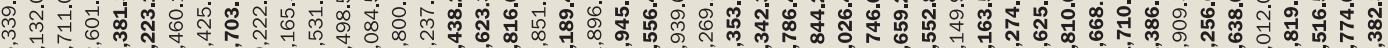

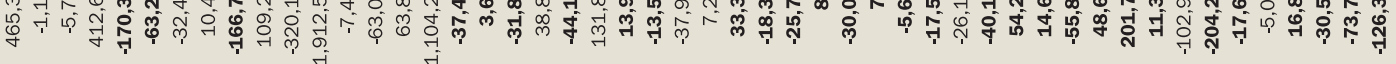

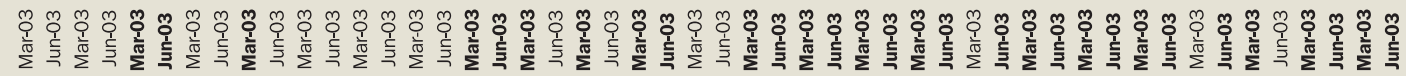

$$
\text { 至 }
$$


Ainda no que se refere à eficácia dos modelos autoregressivos, deve-se mencionar que tais modelos são fortemente baseados em informações passadas, sendo incapazes de prever choques inéditos. 0 modelo proposto, contudo, não exclui a possibilidade de que cenários de estresse sejam inseridos pelos gestores em seu budget plan, numa abordagem semelhante ao stress test empreendido pelas instituições financeiras em adição ao cálculo do VaR.

\section{NOTAS}

${ }^{1}$ Os testes de Breusch-Pagan e Hausman revelaram a existência de efeitos fixos, como esperado, já que as empresas pertencentes à amostra eram bastante díspares entre si. Portanto, a estimação foi realizada considerando-se tais efeitos e extraindo-se uma dummy para cada empresa. Nesta dummy devem estar consideradas todas as variáveis omitidas no modelo.

A pesquisa contou com o financiamento da Capes e do CN Pq, em etapas distintas.

\section{REFERÊNCIAS BIBLIOGRÁFICAS}

BAUMAN, J.; SARATORE, S.; LIDDLE, W. A practical framework for corporate exposure management. In: BROWN, G.; CHEW, D. (Eds.). Corporate Risk: Strategies and M anagement. London: Risk Publications, 1999.

CASTRO, A. Gerenciamento do risco de mercado para produtores de commodities no Brasil. 2002. Dissertação (M estrado profissional em Administração) - FGV-EAESP, São Paulo, 2002.

ENDERS, W. Applied Econometric Time Series. Hoboken, NJ: John Wiley \& Sons, 1995.
FROOT, K.; SCHARFSTEIN, D.; STEIN, J. A framework for risk management. Harvard Business Review, v. 72, n. 6, p. 91-102, 1994.

HAYT, G.; SONG, S. Handle with sensitivity. Risk Magazine, v. 8, n. 9, p. 94-99, 1995.

JP MORGAN \& CO.; ARTHUR ANDERSEN LLP FINANCIAL ENGINEERING LTD. The JP M organ/Arthur Andersen Guide to Corporate Risk Management. London: Risk Publications, 1997.

LAROQUE, E.; LOWENKRON, A.; AMADEO, E.; JENSEN, J. Cenários probabilísticos: conjugando análise de riscos e projeções macroeconômicas. Documento técnico, 2003. Lista de riscos disponível em «http:// www.listaderiscos.com. br>.

LINSMEIER, T.; PEARSO N, N. Risk management disclosure. In: JAMESON, R. (Ed.). Financial Risk and the Corporate Treasury - New Developments in Strategy and Control. London: Risk Publications, 1997.

RISKMETRICS GROUP. CorporateM etrics Technical Document, 1999. Disponível em «ttp://www.riskmetrics.com>.

SHAPIRO, A.; TITMAN, S. An integrated approach to corporate risk management. In: BROW N, G.; CHEW, D. (Eds.). Corporate Risk: Strategies and M anagement. London: Risk Publications, 1999.

SIMS, C. Macroeconomics and reality. Econometrica, v. 48, p. 1-49, 1980.

STEIN, J.; USHER, S.; LAGATTUTA, D.; YOUNGEN, J. A comparables approach to measuring cash-flow-at-risk for non-financial firms. Journal of Applied Corporate Finance, v. 13, n. 4, p. 8-17, 2001.

STULZ, R.; WILLIAMSON, R. Identifying and quantifying exposures. In: JAMESON, R. (Ed.). Financial Risk and the Corporate Treasury - New Developments in Strategy and Control. London: Risk Publications, 1997.

VERMEULEN, E. Corporate Risk Management: A Multi-Factor Approach. Amsterdam: Thesis Publishers, 1994.

\section{Artigo recebido em 10.08.2004. Aprovado em 28.07.2005.}

\section{Fernanda Finotti C ordeiro Perobelli}

Professora da FEA-UFJF. Doutora em Administração pela FEA-USP.

Interesses de pesquisa nas áreas de finanças corporativas e risco.

E-mail: ffinotti@labfin.com.br

Endereço: Rua Antônio Carlos, 440/403, Centro, Juiz de Fora - MG, 36010-560.

\section{José Roberto Securato}

Professor da FEA-USP. Livre Docente e doutor em Administração pela FEA-USP.

Interesses de pesquisa nas áreas de finanças.

E-mail: securato@usp.br

Endereço: Av. Prof. Luciano Gualberto, 908, FEA1, sl. G116, Cidade Universitária, São

Paulo - SP, 09080-500. 